\title{
Co-design at a Distance: Context, Participation, and Ownership in Geographically Distributed Design Processes
}

Dr Cara Broadley, c.broadley@gsa.ac.uk,Dr Paul Smith, p.smith@gsa.ac.uk

\begin{abstract}
This paper aims to understand how effective co-design activities can be carried out with remote and rural communities. Taking a combined ethnographic and participatory approach in our project situated in the Scottish Highlands and Islands, we discuss two iterative phases of scoping, idea generating, and prototyping and refining. Throughout these accounts we reflect on how creative design methods enhanced shared understandings of local contextual issues; drove us to identify people and resources to enrich the participatory design process; and strengthened their ownership over the project. Building on this we propose a series of recommendations for design researchers to stimulate engagement with communities from remote and rural geographies, support their participation in activities that aim to understand past challenges and successes, and enable them to contribute to the design and development of new outcomes through sustained collaboration.
\end{abstract}

Keywords: co-design; participation; distance; context; ownership

\section{Introduction}

Participatory Design (PD) was born in the 1960s from a desire to address power imbalances and regain human accountability in light of technological advancements, and has since been adapted to explore wider social challenges (DiSalvo et al., 2012). Steen (2011: 50) positions PD as a practice in which design researchers apply creative methods to engage with users and stakeholders and build upon their primary knowledge and expertise ('what is') to envisage preferable scenarios ('what could be').

Consequently, the role of the user evolves from a consumer, to a respondent, to a participant, and in some cases, to a co-designer, who actively contributes to the design process and its outcomes (Sanders and Stappers, 2008: 12). 
Exploring factors that constitute effective PD, this paper aims to understand how effective co-design activities can be carried out with remote and rural communities. We begin in section two, Co-design within Participatory Design, by interrogating the notion of participation and how different degrees of involvement and activities can inform distributions of agency and ownership in design. In section three, $A$ Combined Ethnographic Approach, we explain how we combined ethnographic and participatory methods in our approach, operating iteratively through cycles of scoping, idea generating, and prototyping and refining. In section four, Peer-to-Peer Community Engagement, we present an illustrative example from phase 01 of our primary research carried out in the Scottish Highlands and Islands as part of the Leapfrog project.

Leapfrog: transforming public sector engagement by design is a $£ 1.2$ million Connected Communities project funded by the Arts and Humanities Research Council (AHRC). Delivered through a partnership between ImaginationLancaster at Lancaster University and The Innovation School at The Glasgow School of Art, the Leapfrog project is working initially with public sector and community partners in Lancashire and the Highlands and Islands of Scotland to create and evaluate new tools and models of creative engagement. Lancashire has closely packed overlapping communities that are hard to engage, for example, people with low rates of English literacy. In the Highlands and Islands communities are geographically dispersed and often located in remote, hard-to-reach areas, and as such are strongly motivated to actively address the challenges they face in terms of communications and access to, for example, services and resources. Working across these two locations will test new approaches to engagement and help make them more robust when applied in other parts of the UK. These engagement tools will be used by communities directly and will also be exchanged with others who will be encouraged to appropriate and adapt these tools to 
fit their own needs. Tools could be physical, digitally downloaded and printed, or entirely digital in nature (Leapfrog, 2017a).

We return to the field of PD in section five, Reflection and Iteration, to examine how distance (geographical, as well as social and cultural) has been conceptualized and managed, and to frame our reflections on the shortcomings of the co-design activities we carried out. We then explain how we applied a range of creative methods in phase 02 of the project to unpack local needs and aspirations together with our community partners, before co-designing a series of engagement tools as outcomes. Framing our insights as three recommendations for design researchers, in section six, Co-design at the Edge, we highlight the role of visual and reflective practice in stimulating engagement with people in remote and rural geographies; the application of bespoke and flexible methods to mediate social and relational interaction, and thus to support participation; and the use of remote and direct approaches and tools to strengthen and sustain ownership in collaborative design activities. We conclude in section seven, Conclusions, by summarizing the paper's contribution and its value to design researchers working with remote and rural communities.

\section{Co-design within Participatory Design}

As a 'heuristic structure for collaborative design' (Wang and Oygur, 2010: 356), codesign promotes exchange between disciplines, professions, businesses, or neighbourhoods, enables the brokering of knowledge, and operates through iterative cycles of development. Generative in nature, co-design activities can range from 'consultation and information gathering to facilitating people in generating their own ideas and solutions' (Cruickshank et al., 2013: 49). Whilst PD is deemed a research-led orientation in which design researchers gain an insight into participants lives (Steen, 2011: 48), both approaches can be thought of as practices through which 'researchers 
and designers attempt to cooperate with or learn from potential users of the products or services which they are developing. Their goal is to develop products or services that match users' practices, needs and preferences' (2011: 45). Following Dorst's Frame Creation model (2015), critical engagement with peoples' existing situations can illuminate both 'significant influences on their behaviour and what strategies they currently employ', and 'practices and scenarios that could become part of the solution' (2015: 76). Taken together, we position co-design as a stage within the PD process that aims to build on contextual insights to inform the development of new design concepts.

\section{Positioning Participation; Positionality through Participation}

The nature of interaction and the mechanisms by which control and power are distributed remain much contested issues in PD (Vines et al., 2013), with Steen (2013) noting that the quality of participation ranges from 'superficial "hand-holding" initiatives to organizing productive dialogue and intimate cooperation' (2013: 949). Recognizing that the extent to which participants inform or make design decisions can fluctuate, Andersen et al. (2015) frame the balance of agency between design researchers and participants in PD as an emergent matter of concern, which is 'intimately intertwined with all sorts of formative and situated relations' (2015: 257). As such, the practical and ethical dimensions of building relationships with individuals and communities underlines the need for design researchers to carefully choreograph their integration of contexts, participants, and methods (Brandt et al., 2012).

\section{Contextual methods for understanding, articulating, and imagining in PD}

Design researchers working in PD employ creative and generative methods including collaging, sketching, 3D modelling tasks, prototypes, and design games as ways of telling, making, and enacting to envisage the future (Brandt et al., 2012). 
Misrepresentation, cultural sensitivity, and the appropriateness of PD methods are amongst the barriers and hurdles awaiting design researchers (Robertson and Wagner, 2012). In response, design toolkits can be seen to advocate the use of ethnographic practices to gain an understanding of environments, behaviours, and situations (IDEO, 2002). Evoking concepts of cultural probes (Gaver et al., 1999), self-documentation is explicated as a technique to learn about participants' behaviours and needs by interpreting their photographs, drawings, and written notes. Established tools including user personas, scenarios, and stakeholder maps (Hanington, 2003; Hanington and Martin, 2012) aim to create visual and textual representations of people within the design context, describing their experiences and aspirations and depicting their interactions within existing and speculative social networks. These premises and practices inform the approach taken within our work in the Highlands and Islands of Scotland as part of Leapfrog.

\section{A Combined Ethnographic and Participatory Approach: Scoping, Idea}

\section{Generating, Prototyping and Refining in Participatory Action Research}

Halse and Boffi (2014) maintain that where ethnography is appropriated by design disciplines, the 'core ethnographic aspects of empathy, open-endedness, attentiveness to situatedness, have met with designerly competencies' (2014: 4). Adopting this ethos is critical to a PD practice that is both socially inclusive and responsive to local capabilities, strengths, and resources (Smith and Broadley, 2016), and as Manzini sets out, products, services, and models that address societal needs and foster new collaborations 'emerge from the creative recombination of existing assets (from social capital to historical heritage, from traditional craftsmanship to accessible advanced technology), which aim to achieve socially recognized goals in a new way' (2015: 11). 
Developing a combined participatory and ethnographic approach throughout our work, we blend creative methods from across PD to both enrich our own knowledge of established contexts and situations, and encourage communities to share their experiences and envisage the future together.

Drawing from Participatory Action Research (PAR) (Chevalier and Buckles, 2013), the project discussed in this paper is framed through two phases of scoping, idea generating, and prototyping and refining. Centered on the relationships formed between researchers, practitioners, communities, and organisations, PAR draws from experiential learning and domain-specific expertise to offer a framework for conceptualizing new knowledge within research contexts (Howard and Somerville, 2014). Whilst originating from the field of organizational behaviour and prevalent across the social sciences, PAR is often adopted in PD studies, in which the design process entails iterative cycles of planning, acting, observing, and reflecting in response to dialogue and interaction (Howard and Somerville, 2014; Bilandzic and Venable, 2011). A flexible PAR framework supported us in bringing together a team of design researchers, regional development coordinators, community development practitioners, and people from local groups and organisations to identify past challenges and barriers around community engagement and develop new approaches.

\section{Peer-to-Peer Community Engagement: Co-Designing Engagement Tools with}

\section{Remote and Rural Communities}

The Peer-to-Peer Community Engagement project aimed to work with remote, rural, and dispersed communities in the Scottish Highlands and Islands. Its aim was to codesign a series of engagement tools to support community practitioners to connect and interact with a broader range of people from local organisations and groups, and to 
conduct quality peer-to-peer consultations to inform the planning and delivery of future initiatives across the region. The project took place in two locations in the west of Scotland: the Isle of Mull in the Inner Hebrides and the Kyles of Bute in the Cowal Peninsula. Both locations are respectively 102 and 80 miles from the city of Glasgow, where our design team were based, and travel to these locations involves train, car, and often ferry journeys. Working with a regional development agency as gatekeepers, we connected with development trusts - community-owned organisations that seek to enhance and support local initiatives by addressing a range of economic, social, environmental and cultural issues (DTA Scotland, 2017) - in both locations as project partners.

\section{Phase 01}

In the scoping stage we conducted semi-structured telephone interviews with members of development trusts to gain an insight into prior and current community projects, community involvement in consultation, and potential local groups we could collaborate with. Upon meeting community members in person, we used an Individual Mapping Tool, shown in Figure 1 as a graphic template to explore community members' connections to each other by encouraging their annotations and reflective discussion; and a Network Mapping Tool, shown in Figure 2, which through attaching tags and building relational threads, elicited their understandings of how different groups cluster around shared purposes, motivations, resources, and outcomes.

In the idea generating stage participants leading local projects worked in teams to define people they deemed hard-to-reach whilst we captured their experiences of previous engagement initiatives on sticky notes to create an archive of past successes and failures. Figure 3 shows how we thematically mapped (Braun and Clarke, 2006) key 
concepts from participants' insights into a set of shared principles for local engagement and began to consider how these could be embodied in a series of co-designed tools.

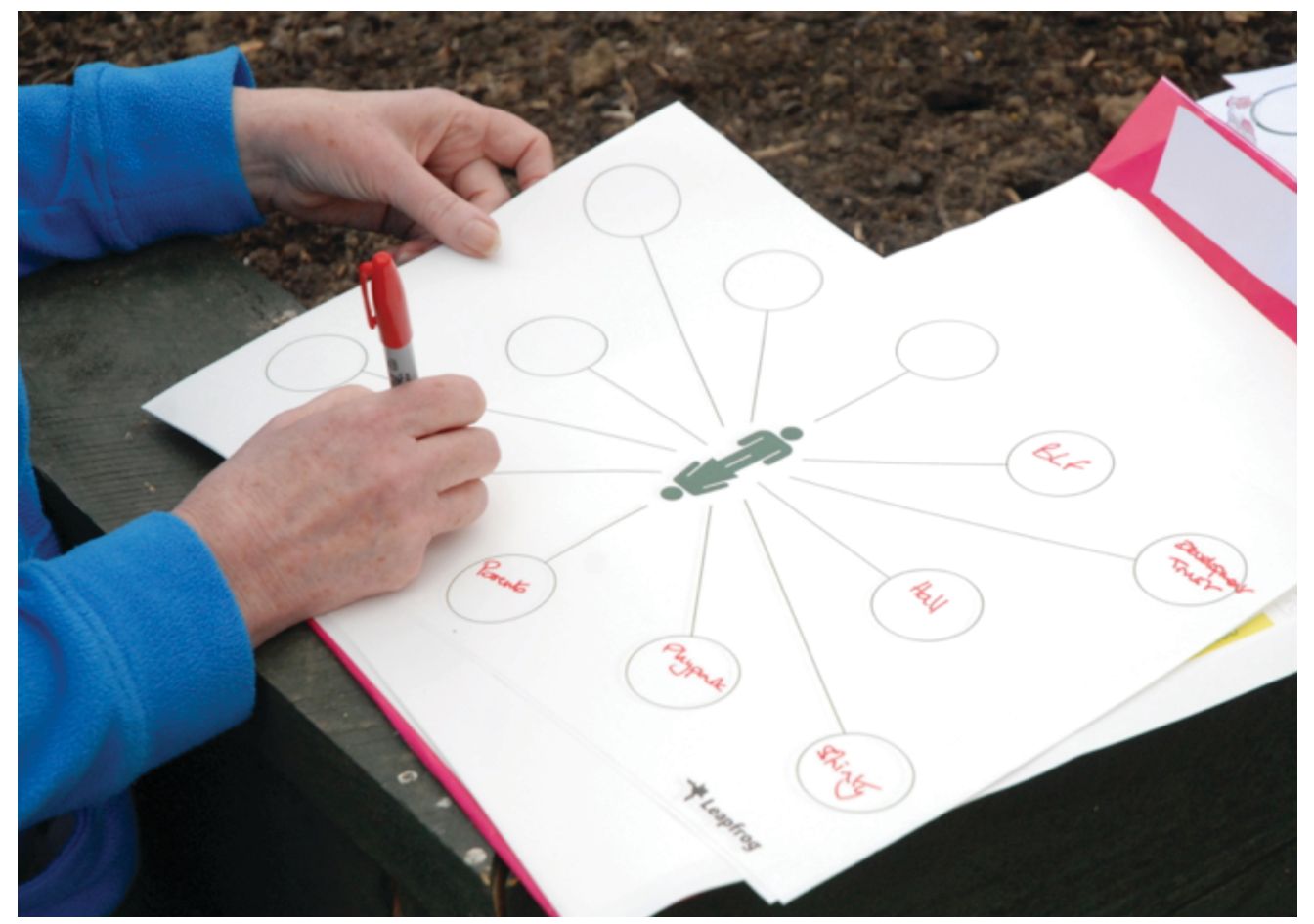

Figure 1: The Individual Mapping Tool. Photograph courtesy of Authors.

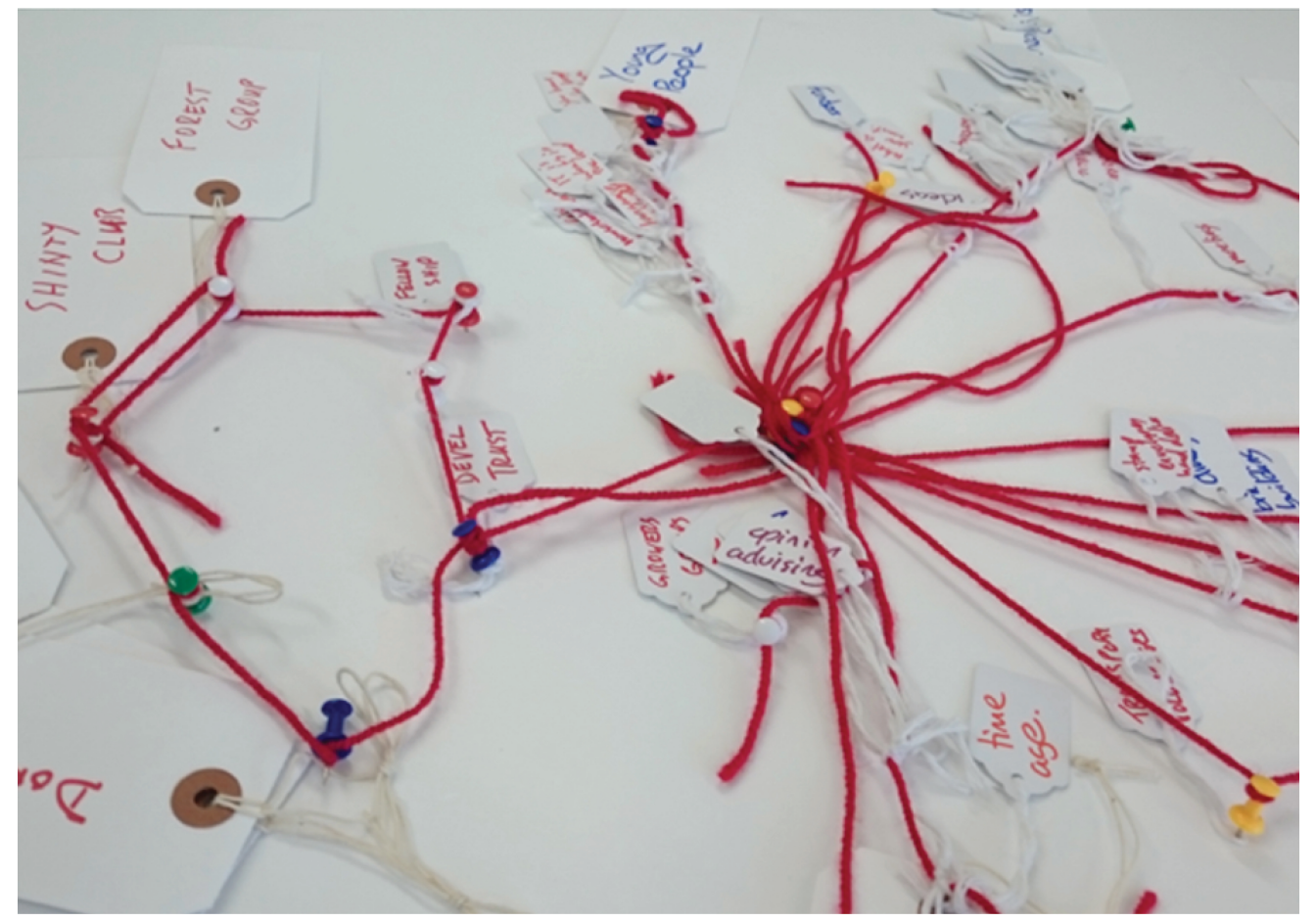

Figure 2: A completed Network Mapping Tool. Photograph courtesy of Authors. 
In the next stage the groups sketched out their ideas before presenting initial design concepts, voting for their favourites, and ranking and prioritizing those to develop. Engagement tool ideas included a set of playing cards to creatively develop ideas for future projects and events, a template form to quickly plan a community event, small paper tickets to prompt public feedback on local issues, a newspaper tool to collate and broadcast community-led stories, and customizable food packaging to promote local projects

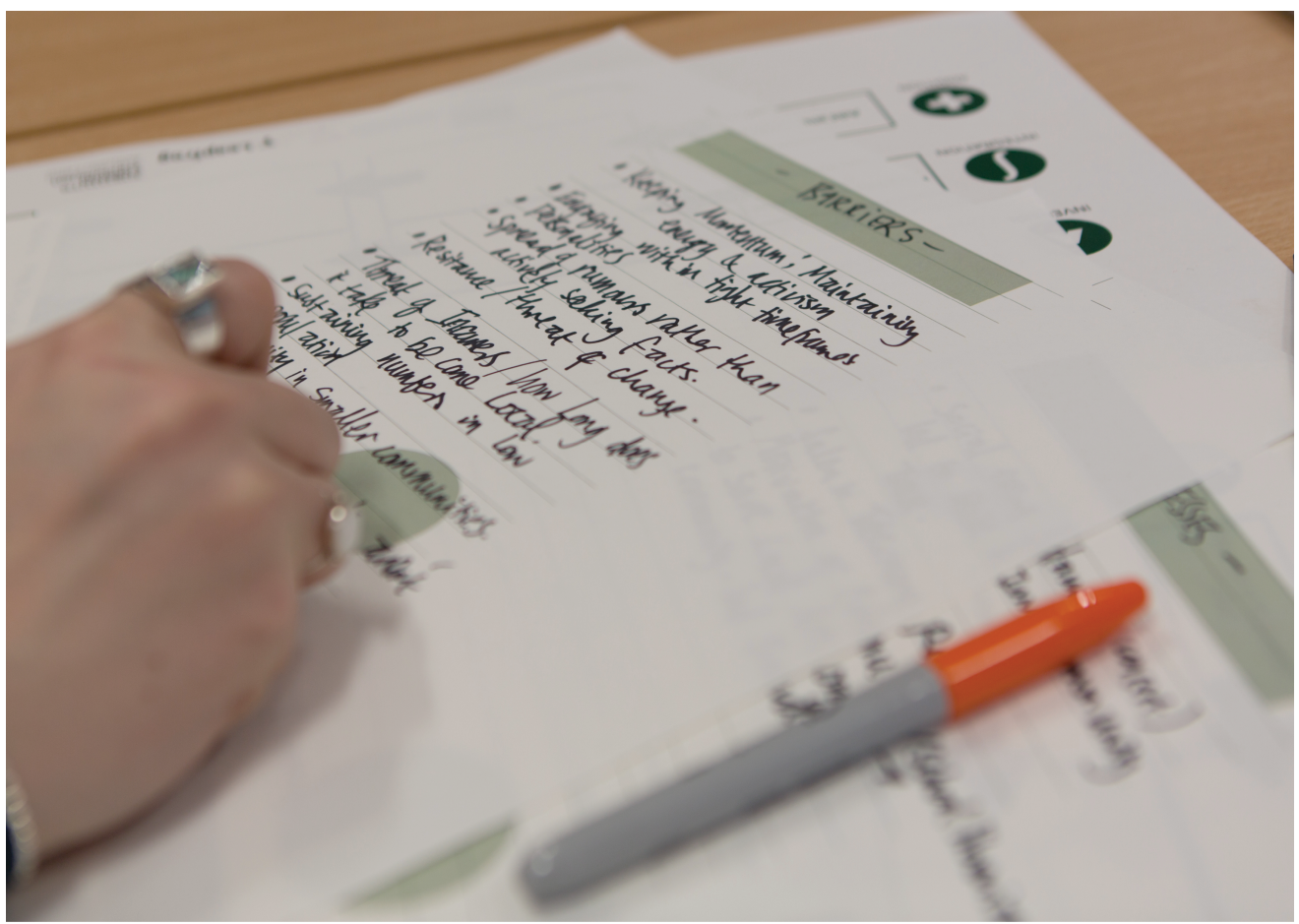

Figure 3: Collectively consolidating barriers and successes of community engagement. Photograph courtesy of Authors.

In the prototyping and refining stage we worked with participant groups to test paper mock-ups of the engagement tools and adjusted the designed elements and textual content in order to enhance their functionality. 


\section{Reflection and Iteration: Distance as a Barrier and an Opportunity to Engagement, Participation, and Co-design}

Noting a gap in the surrounding literature regarding the explicit discussion of geographic distance as a factor that can limit engagement, participation, and co-design activities, we conceptualize social and cultural difference as a broader set of circumstances and intrinsic element of distance that has come to characterize both the rationale for and the means by which many PD initiatives are carried out. Factors contributing to a sense of distance between design researchers and communities can include differences in age, ethnicity, race, religion, class, language, disability, gender, and sexuality, and each of these can be seen to play a central role in determining the character of the project or intervention. In spite of disciplinary developments in PD, the practice originates from a determination to advance a democratic and emancipatory ethos by involving people who may have been marginalized by mainstream society and who can be considered in some way distant from the design process (Robertson and Simonsen, 2012; Björgvinsson et al., 2012). Developing our reflections from phase 01 of the project, we highlight the notion of distance as a significant barrier to engagement for us, and a foundation on which to build co-design partnerships with the communities.

\section{Scoping with Publics to Engage with Contextual Issues}

By working primarily with the development trusts in defining the project aims, we inadvertently excluded the experiences and perspectives of people leading local projects from the scoping stage. Engaging with publics constituted by their connections to a shared issue (Ehn, 2008; Björgvinsson et al., 2012), community-based PD focuses on building networks of individuals that may not otherwise exist as a cohesive entity and is mindful of the 'social constructs and relations' that characterize and permeate these groups (DiSalvo et al., 2012: 183). In employing the Individual Mapping Tool and 
Network Mapping Tool with a range of such stakeholders, we illustrated key individuals, their level of current activity in the community, and their perceived importance. However, in broadly depicting fragments of these landscapes, this method did not support us to deeply interrogate grass-roots local concerns together or identify opportunities for transformation. This highlights the need for PD methods and practices to explicitly recognize and integrate existing and prospective publics, to account for corresponding relational factors when establishing partnerships with community groups, and the need to move from a holistic consideration of the surrounding social, cultural, and geographic context towards specific issues efficiently and inclusively.

\section{Developing Shared Understandings and Aspirations as a Springboard for Idea}

\section{Generating}

An initial focus on past community engagement challenges, barriers, and failures inhibited the groups' abilities to engage in collaborative idea generation. With this informal conversation occurring by default rather than design, we adapted our workshop plan to synthesize insights and emergent themes. Symptomatic of the lack of granularity achieved through the scoping stage, this suggests that targeted methods to enhance the prioritization of issues and frame co-design activities can enhance participants' ownership over and motivations to address local challenges. At the same time, a commitment to 'getting to know each other' can align the aims and aspirations of design researchers and participants (Fox and Dantec, 2014: 787), and openness, flexibility, and adaptability can initiate community involvement and provide 'meaningful communication, so that social relationships are maintained' (Branco et al., 2017: 128). Reflecting on our use of generalized co-design approaches in phase 01 , this leads us to consider how, in developing methods that respond to local issues, design researchers can support community members' imaginative abilities, expose 'novel resources and 
opportunities to reconsider their environment' (DiSalvo et al., 2012: 196), and 'promote the growth of diffuse collaborative design capabilities' (Manzini, 2015: 154) in response to their social, cultural, and geographic circumstances.

\section{Situating Co-design Outwith the Workshop}

Our lack of direct interaction with participant groups between the workshops prevented their involvement in design decisions. Working independently, we applied graphic techniques to filter and interpret insights into prototype engagement tools, and fundamentally, our relationship as a co-design team was fractured. Building on the cultural probe approach and a multitude of reappropriations and critiques it has informed (Gaver et al., 2004; Mattelmäki, 2006), our research has previously defined remote probes as tools given to participants to complete in the absence of the design researchers (Broadley and McAra, 2013; Broadley, 2013), whilst a direct probe denotes an artefact deployed in a workshop or interview setting. Combinations of remote probes and direct probes can enrich engagement by contextualizing interactions and fostering a sense of mutual understanding, yet our interactions with participants were bounded by the workshop experience. Whilst we left them with abstract concepts for future development once we departed, we overlooked opportunities for prompting and provoking ongoing creative thinking.

These reflections set the scene for us to describe our approach in phase 02 of the project and the steps we took to negotiate and capitalize on aspects of distance in codesign activities.

\section{Phase 02}

Challenges around the planning, delivery, and sustainability of community-led events emerged as a core insight from participant groups in phase 01 of Peer-to-Peer 
Community Engagement. This provided an opportunity to focus in the scoping stage of Phase 02 on practical and logistical challenges and gaps from within event planning processes and to co-design tools to address unmet needs. Working with a Local Area Development Officer we recruited individuals from social businesses and community enterprise representatives with an interest in event planning and engaging with local people about their services.

In the workshops we carried out an icebreaker in which the participants and the design team individually completed a Profile Card to identify their individual skills, capabilities, or superpower in event planning, and to consider their weaknesses or nemesis.

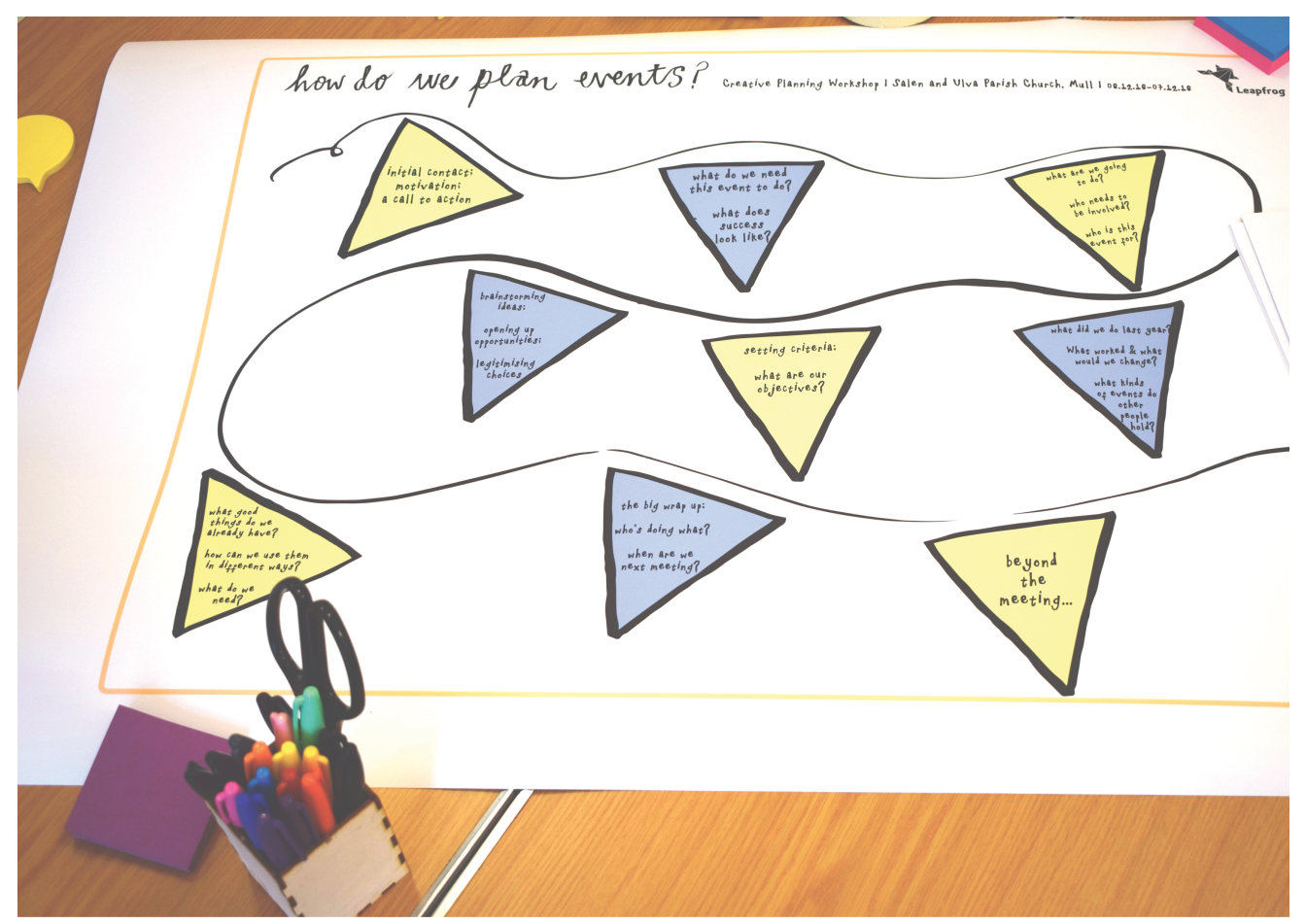

Figure 4: The Journey Map tool. Photograph courtesy of Authors.

In preparation for the workshop we created a Journey Map tool, shown in Figure 4, depicting a sequence of stages from initial strategic discussions around an event's purpose, to distributing roles and allocating tasks, to reflecting on previous event 
successes, to generating new ideas. Encouraging the participants to reflect on their parallel experiences, we verbally shared an anecdotal account of the challenges that we have encountered when progressing through these stages.

Positioning the Journey Map as an underlay in Figure 5, we invited participants to include additional elements of their own event planning processes, and to add depth regarding specific challenges. Using We Need cards shown in Figure 6, participants suggested opportunities such as an accessible inventory of local people and resources, and ways to promote their projects at existing events.

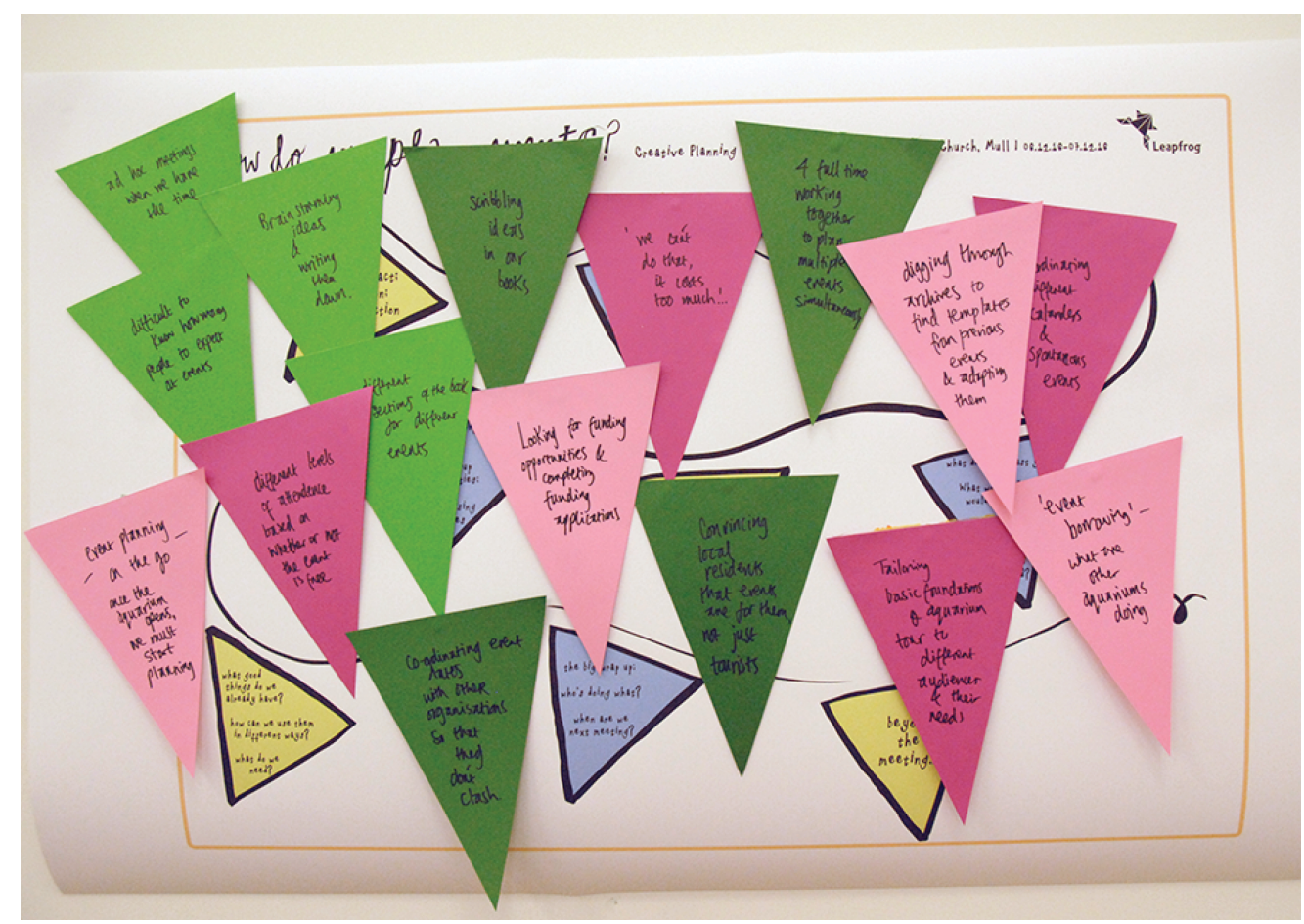

Figure 5: Adding detail to the Journey Map tool in response to Mull workshop participants' experiences of event planning. Photograph courtesy of Authors. 


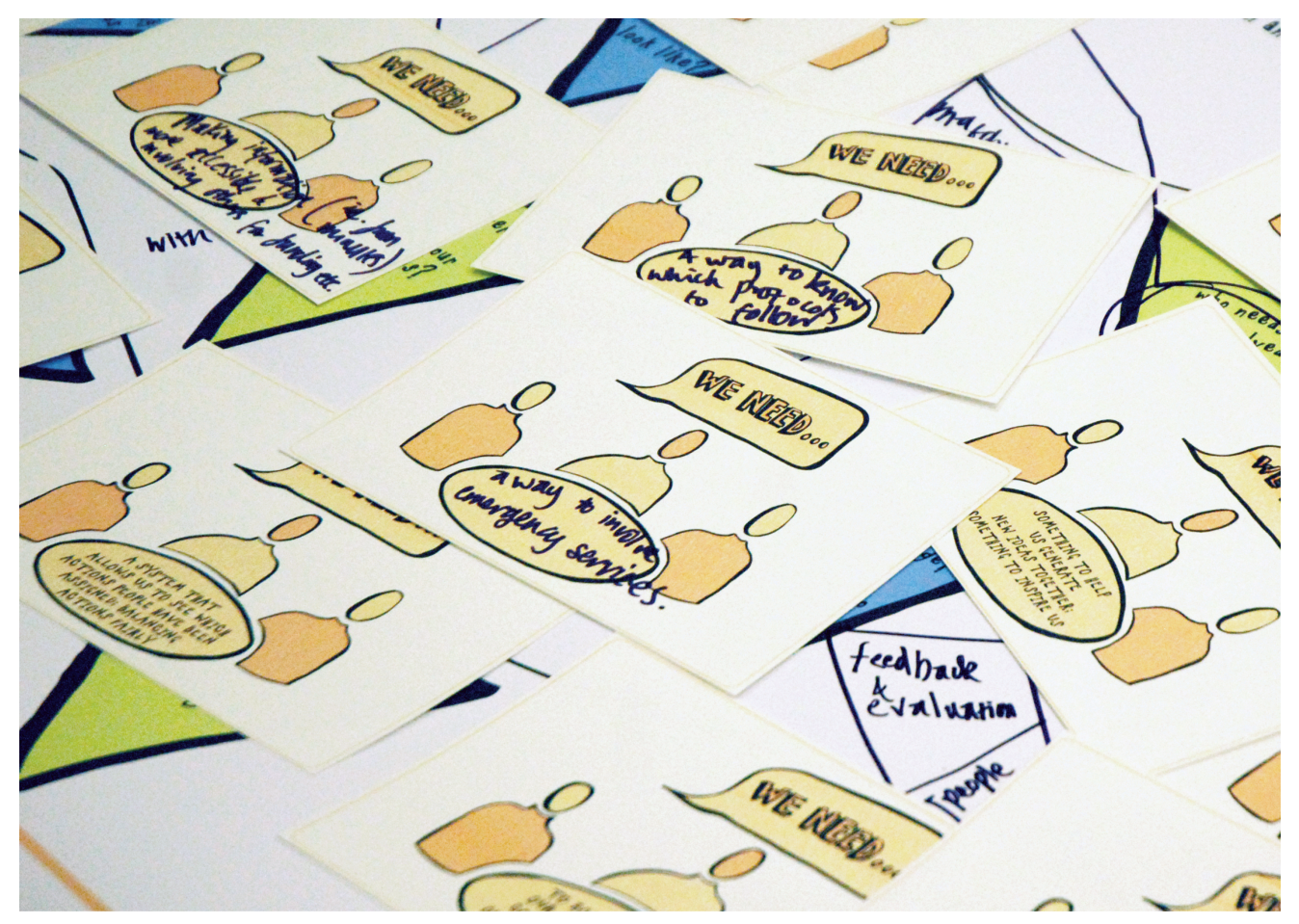

Figure 6: Examples of the We Need cards, used with the Journey Mapping Tool to explore opportunities for tools. Photograph courtesy of Authors.

Our objective in the idea generating stage was to define how the community event planning process could be supported and enhanced. With the intention of articulating the relationship between identified needs and the capabilities of co-designed tools to support community events, we introduced two outcomes of the phase 01 workshops the Raffle Ticket and Creative Thinking Cards - as shown in Figure 7 and Figure 8 - as exemplar tools to inspire a brainstorming activity. 


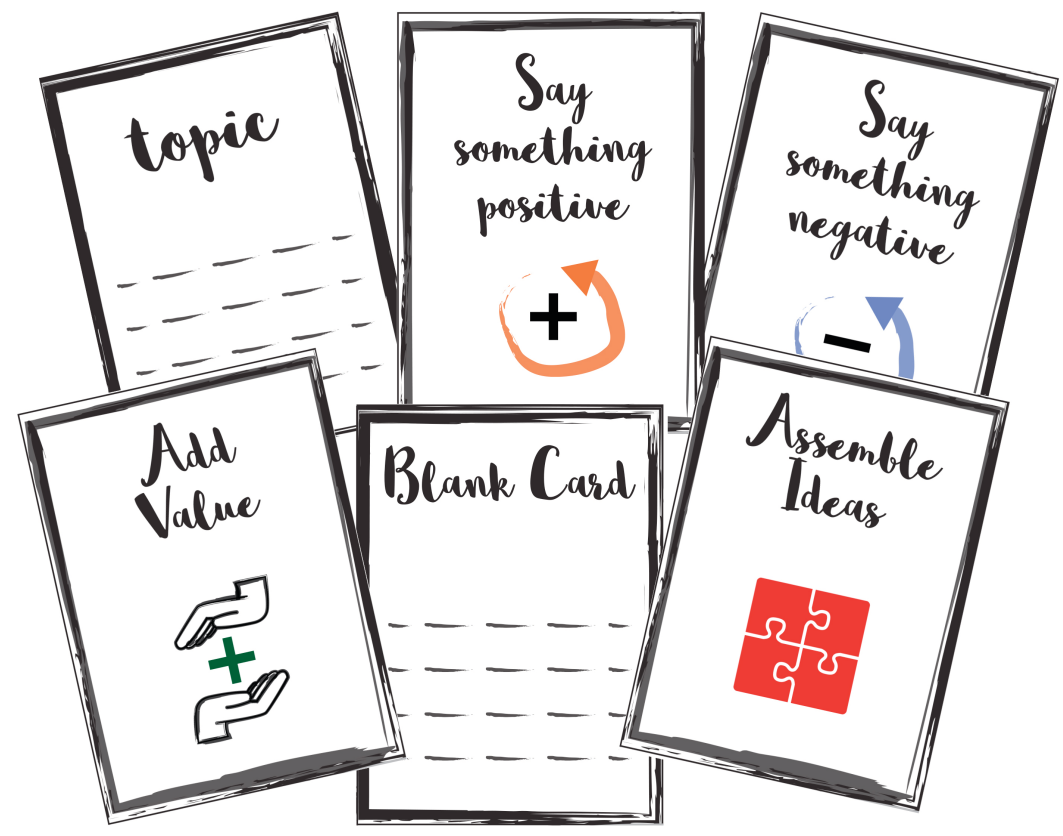

Figure 7: Creative Thinking Cards - prototype tool concept for reflecting on previous events and collectively generating ideas for new initiatives. Photograph courtesy of Authors.

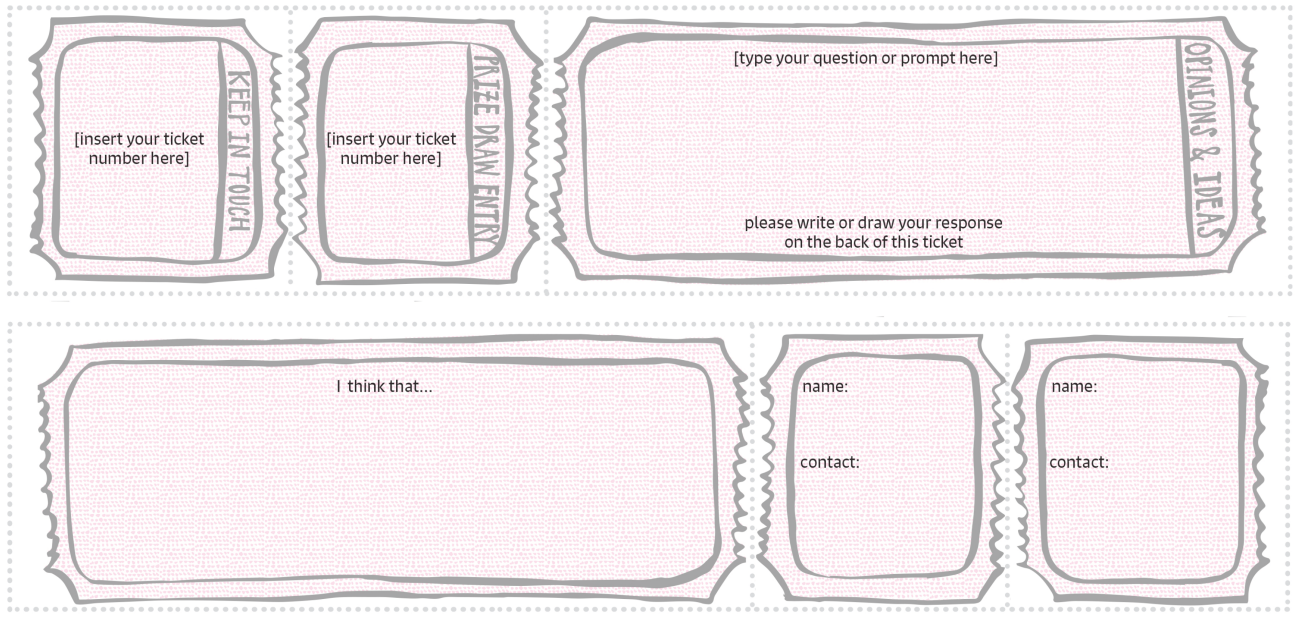

Figure 8: Raffle Ticket - prototype tool concept for collecting community responses to local issues. Photograph courtesy of Authors.

This led us into a prototyping activity in which participants selected ideas to take forward before constructing mock-up tool concepts. Figure 9 shows a group of participants developing their vision for a portable event planner and an adaptable 
system of codes and symbols that allows users to capture information, insights, and reflections at each stage of the process.

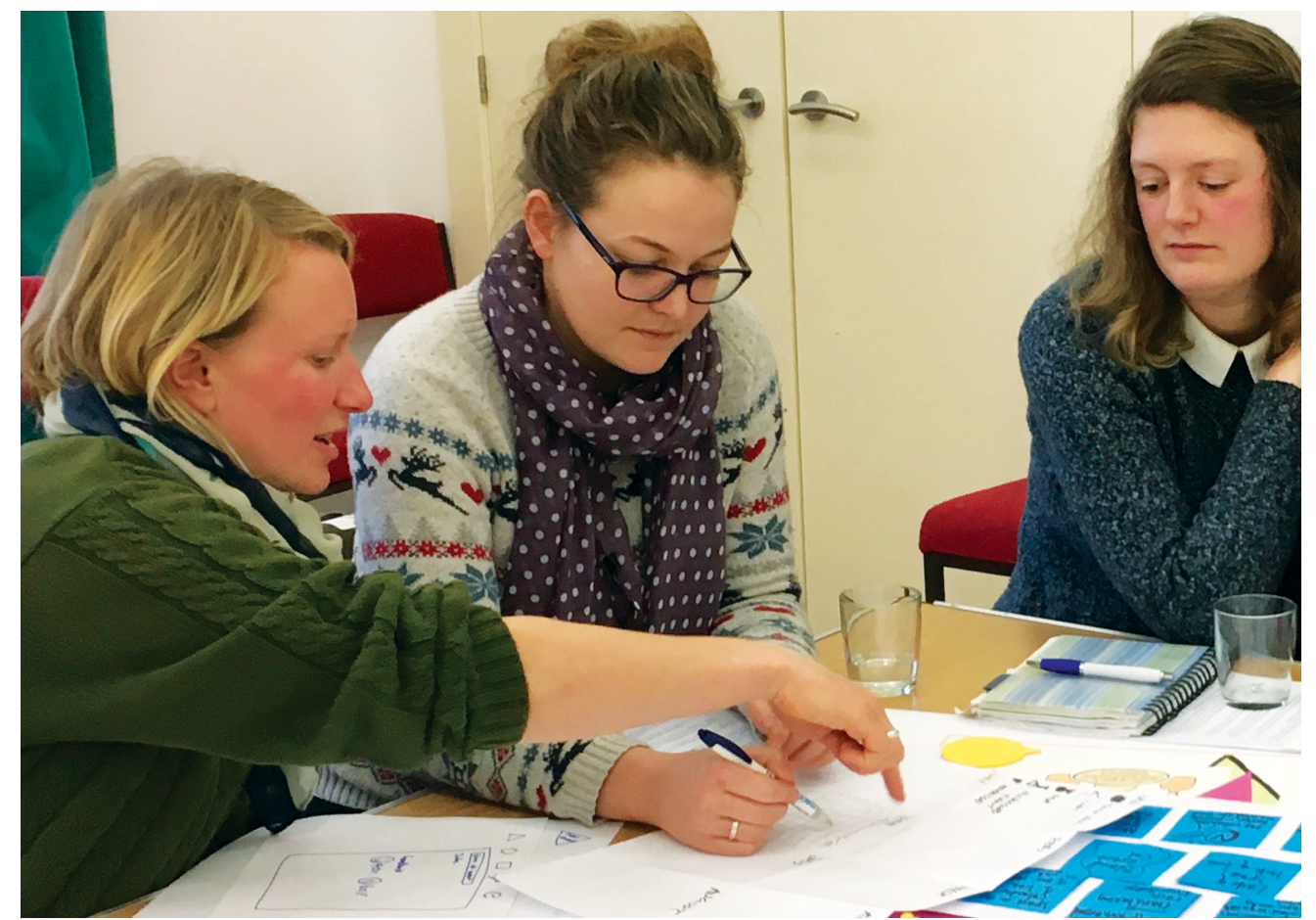

Figure 9: Mull workshop participants. Photograph courtesy of Authors.

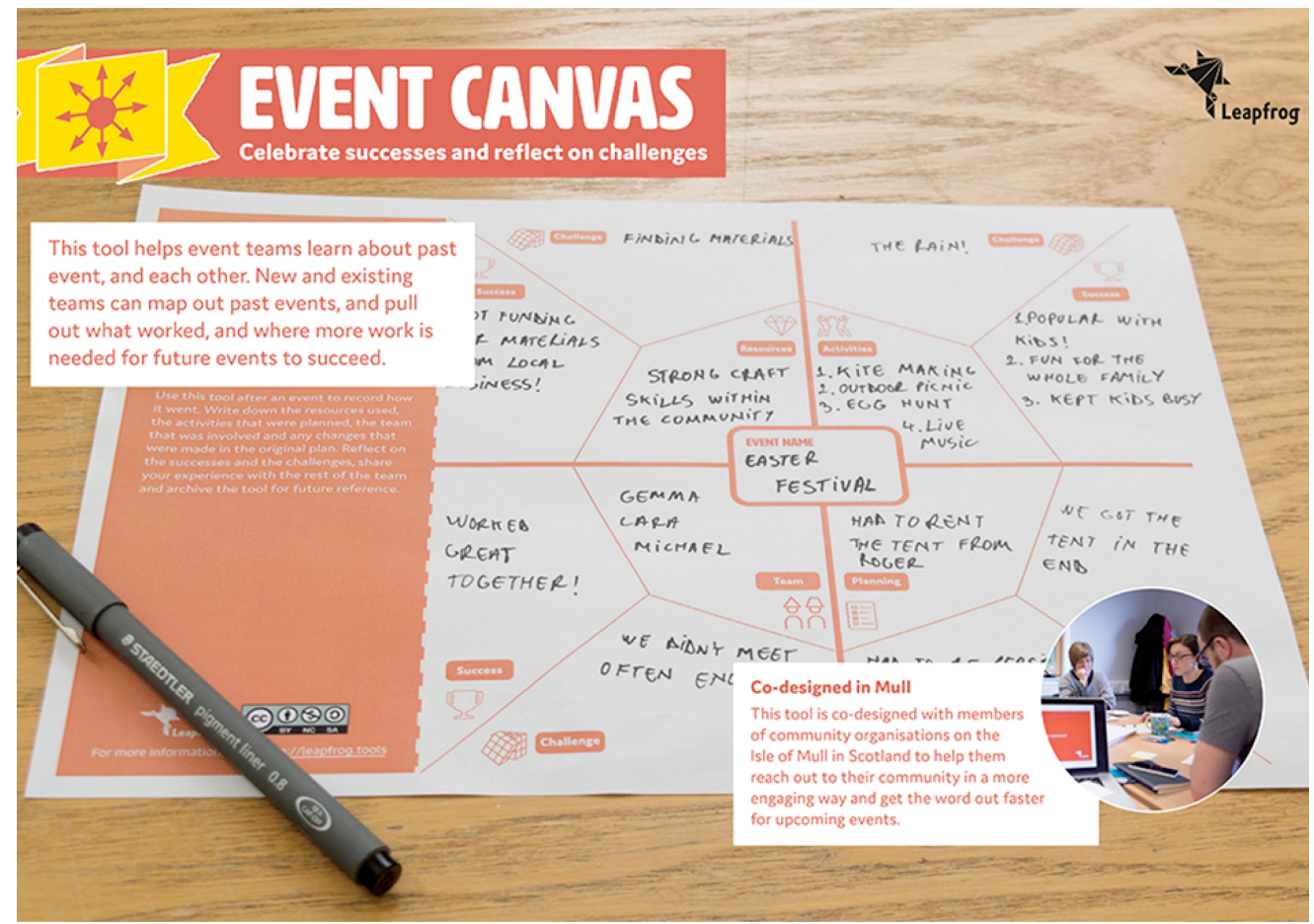

Figure 10: The Event Canvas tool. Photograph courtesy of Authors. 
We left these prototypes with the participants in Mull whilst we refined the detail surrounding their features and functionality remotely. Our development of the event planning tools was strengthened through contributions from a core participant group via frequent email contact, digital feedback sheets, and video conferencing sessions. This phase culminated in the finalized tools being disseminated to a wider group of volunteers, community development practitioners, and social enterprises at a celebratory event in the highland region. Figure 10 shows one of the co-designed tools the Event Canvas - as a published co-designed outcome from Peer-to-Peer Community Engagement (Leapfrog, 2017b).

\section{Co-design at the Edge: Stimulating Engagement, Supporting Participation, and Enabling Collaboration}

Drawing from both phases of Peer-to-Peer Community Engagement, we now discuss how elements of our ethnographic and participatory approach supported our choreography of the PD process in a rural context and helped us to remotely sustain a critical dialogue with the participants. Framing our insights as a set of three recommendations for design researchers, we affirm the role of visual and reflective practice in stimulating engagement with people living and working in remote and rural geographies; the application of bespoke and flexible methods to mediate social and relational interaction, and thus to support participation; and the use of remote and direct approaches and tools to strengthen and sustain ownership in collaborative design activities.

\section{Visual and Reflective Practice to Understand Context}

Whilst we agree that in PD there is a need to express 'a mutual understanding which places the stakeholder at the centre of the engagement strategy' (Chamberlain et al., 2013: 137), we concur with Manzini and Rizzo's view (2011) that as facilitators, 
mediators, and triggers, (2011: 2013) design researchers' conceptual and material practices influence the social dynamics of group relations and have a tangible impact on project processes and outcomes. The emergence of 'knowledge gaps between designers and community members, binding local socio-cultural protocols, and tensions between the designers' and local epistemological frames' (Sabiescu et al., 2014: 1) present significant challenges in community-based PD, and reinforce the need for activities that account for the distinct and overlapping concerns, insights, and ideas of external design researchers and local communities. Immersing themselves in the investigative context and dedicating time and space to develop trust with groups allows design researchers to unite with local gatekeepers to broker buy-in from the wider community (SsoziMugarura et al., 2017). Whilst this can be difficult to attain due to logistical and resource issues, we have found that visual 'design' tools can support the process.

In examining the often "“invisible" work of engagement' in PD through their work with rural communities, Chamberlain et al. (2013) maintain that new practices are instilled and embedded via 'negotiations with a series of stakeholder communities in the design setting and ethnographic understandings of the site and community' (2013: 131). In phase 02 our preparation and coordination of the Journey Map instigated open-ended informal dialogue with the participants and promoted a sense of conviviality and rapport. By making our knowledge transparent through its visual nature and verbally relaying a contextual example of the process in action, we established a shared understanding of current barriers and challenges.

As surfaces upon which contrasting and parallel experiences and insights could be accumulated, the Profile Cards and Journey Map revealed each participant's event planning skills and perceived weaknesses and emphasized their central roles in shaping and refocusing the co-design activities. As Danish designers working with people living 
in rural India to create a waste management system, Vestergaard et al. (2016) observe how personas 'created a substantial understanding of the users' individual needs, interests, values and emotions and helped to overcome the physical and cultural distance, enabling a strongly contextualised design' (2016: 257). Whilst stimulating empathy and strengthening collaborative bonds, our methods enabled orientation amongst the group and illuminated authentic contextual issues as the foundations of the co-design activities in the idea generating and prototyping and refining stage.

This underlines the design researcher's responsibility to carefully coordinate sustainable engagement approaches through orienting and embedding themselves in the social, cultural, and geographic context. As an iterative act of inquiry (Schön, 1983), reflective practice can mediate such barriers (Emilson et al., 2014) and stimulate openended discussion with participants, reframing current situations, unpacking and interrogating insights that emerge, and applying this experiential knowledge to inform and shape co-design activities. Just as local traditions, precedents, and cultural expressions permeate communities' perceptions of participation, design researchers bring with them their own 'understandings and experiential luggage' (Sabiescu et al., 2014: 8). In calling for approaches to render such knowledge transparent and tangible, we advocate reflective practice as supporting the development of engagement strategies in community-based PD that recognize multiple intersubjective perspectives of participation.

\section{Bespoke and Flexible Methods to Mediate Social and Relational Interaction}

Elaborating on Arnstein's model of civic engagement (1969), Munoz et al. (2014) characterize five distinct levels of participation from attending community events, to being an active member of local organisations, to taking part in community projects, to joining a committee, to playing a key role in organising new services, and conclude that 
commitment at the top end of this scale is strong but restricted to a relatively small group in rural communities. Whilst not concerned with enhancing community involvement in PD processes, this does reinforce both the challenges and potential of galvanizing participants in order to move towards stages of idea generating. In response, the We Need cards enabled the participants to revisit the challenges they had externalized and overlaid onto the Journey Map and to embark upon a further level of critical reflection around their requirements underlying each stage of the event planning process. Emerging from our own insights as bespoke tools, the We Need cards were translated into generalized prompts for supporting participants to share their situated experiences and aspirations and encourage individuals from different organisations and groups with diverse agendas and objectives to locate similar concerns and shared interests.

Individuals residing in the same geographical location may contain several distinct or overlapping subgroups and possess divergent values and ideals. At the same time, communities can form and thrive around shared identities, interests, and practices (DiSalvo et al., 2012), and as such, methods and approaches for supporting their participation in design processes must be created with these contextual factors in mind (Winschiers-Theophilus, et al., 2012; Robertson and Wagner, 2012). The challenges elucidated by the participants centred around practical concerns such as storing and accessing event planning information, involving people with the relevant skills and attributes, and determining contingency plans from the offset of community projects. Using the Journey Map to materialize such issues supported people from different organizations and often with contrasting objectives to coalesce around shared concerns. At the same time, the We Need cards allowed us to capture and curate a repository of contextual insights and opportunities through which to anchor the PD process. By 
encouraging participants to explore their skills and talents and 'a community's particular knowledge and desires' (DiSalvo et al., 2012: 196), community-based PD can instill a sense of pride, agency, and responsibility. When working at a distance, we propose that bespoke and flexible methods support participants to contribute to the process and strengthen their collective articulation of guiding principles to underpin prototyping and refining as co-design activities.

\section{Remote and Direct Approaches to Strengthen and Sustain Ownership}

As DiSalvo et al. recognize (2012), there is an increasing need for portable and mobile co-design activities capable of travelling across social and cultural divides and encompassing the multiple and mutable roles and responsibilities of all individuals involved in the process. Acknowledging the challenges of sustaining productive design relationships once onsite-fieldwork comes to a close, 'the lack of inhabitants' involvement in the final design phase creates certain limitations in terms of predicting a successful product' (Vestergaard et al., 2016: 272), as well as curtailing the participatory and democratic ethos of the project. Reiterating these concerns through their explorations of co-design with older people, Botero and Hyysalo (2013) suggest that 'more sustained and open design strategies' can strengthen and sustain participants' ownership over project processes and reinforce their roles as collaborative partners and key contributors to the designed outcomes (2013: 50).

Marking a notable shift in the workshop towards the prototyping and refining stage, this recalls a critical incident in which participants autonomously sketched the event planning calendar and enacted its functions and features to the wider group. Developing Chamberlain et al.'s (2013) use of design concepts to elicit discussion and evaluation with local stakeholders, the focus of the prototyping and refining stage in phase 02 involved finalising the event planning tool concepts at our base before posting, 
emailing, and presenting these back to participants via video conferencing calls. These paper-based artefacts took on the role of mock-ups of the project outputs, and engagement tools in themselves. In critiquing their content, format, and visual style, the prototypes were written on, drawn over, torn up, and reassembled by the participants and design team. Building on Botero and Hyysalo's (2013) considerations of developing extended and evolutionary design collaborations and returning to the premises of remote probes (Broadley and McAra, 2013; Broadley, 2013) and direct probes, we position the mock-ups as remnants of co-design activities, which, when left behind in situ, reinforced participants' collaborative mindset and instilled further ownership in the design process and its outcomes.

We recognize that sustaining the commitment of participants from remote and rural settings requires a nuanced range of methods and approaches that account for limitations in access to resources. Munoz et al. (2014) concede that in more remote areas participation rates are observed to be stronger and opportunities to develop innovative approaches to alleviate barriers to service production tend to be more profuse, thus suggesting that 'different strategies might be required to mobilize coproducers in different settings' (2014: 219). We maintain that in order to embark on codesign activities authentically and with integrity, design researchers must first develop responsive and resonant ways to stimulate engagement and support participation (Broadley et al., 2016), for it is only through transcending these necessary acts that contextual understandings are constructed, issues are surfaced, relationships are formed, skills and capabilities are realized, a culture of imagination and experimentation is fostered, and the foundations are laid for developing effective design interventions together. 


\section{Conclusions}

Drawing from an illustrative example of our research within the Leapfrog project, in this paper we have explored how effective co-design activities can be carried out with remote and rural communities. Operating through an iterative PAR framework, we align our combined ethnographic and participatory approach with that of PD's emphasis on engaging individuals and communities, generating collective understandings of existing situations, foregrounding skills and capabilities, and together envisaging and codesigning alternative and preferable future scenarios. Our descriptive accounts of and reflections on Peer-to-Peer Community Engagement accentuate the limitations and shortcomings of our approach in phase 01 , and articulate how these were mediated and managed in phase 02 . This highlighted the role of visual and reflective practice in stimulating engagement with people living and working in remote and rural geographies; the application of bespoke and flexible methods to mediate social and relational interaction, and thus to support participation; and the use of remote and direct approaches and tools to strengthen and sustain ownership in collaborative design activities. At the same time, in interrogating the notion of participation and the extent to which different degrees of involvement and activities can inform distributions of agency and ownership in design, we have both noted a gap in PD literature regarding the explicit discussion of geography as a limiting factor, and conceptualized difference social and cultural, as well as geographical - as a broader and more prevalent set of circumstances and intrinsic element of distance that has come to characterize both the rationale for and the means by which many PD projects are carried out. In this sense, we foresee our recommendations being of value to design researchers seeking to build collaborative partnerships with individuals and communities across a geographical distance, and contributing to higher level debates surrounding the ethical, political, and 
practical concerns of negotiating agency and ownership when working with groups perceived to be marginalized from mainstream society.

\section{Acknowledgements}

We thank Highlands and Islands Enterprise for their support of Leapfrog and the project participants from the Isle of Mull and the Kyles of Bute for their contributions to the co-design workshops and tool development. We also thank Professor Leon Cruickshank, Professor Tom Inns, Elizabeth Brooks, and Madeline Smith for their guidance and feedback. Leapfrog is funded by the Arts and Humanities Research Council (AHRC). We gratefully acknowledge their support.

\section{References}

Andersen, L. B., P. Danholt, K. Halskov, N. B. Hansen, and P. Lauritsen. 2015. 'Participation as a matter of concern in participatory design.' CoDesign: International Journal of CoCreation in Design and the Arts 11 (3-4): 250-261.

Arnstein, S. R. 1969. 'A ladder of citizen participation.' Journal of the American Institute of planners 35 (4): 216-224.

Broadley, C. 2013. Visualising human-centred design relationships: a toolkit for participation. $\mathrm{PhD}$ diss.

Broadley, C., and M. McAra. 2013. 'Making, using and interpreting design probes: how subjective is participation.' Proceedings of the DRS CUMULUS 2nd International Conference for Design Education Researchers: 1432-1452.

Bilandzic, M., and J. Venable. 2011. 'Towards participatory action design research: adapting action research and design science research methods for urban informatics.' The Journal of Community Informatics 7 (3). http://cijournal.net/index.php/ciej/article/view/786/804?utm source=twitterfeed\&utm medium $=$ twitter 
Björgvinsson, E., P. Ehn, and P. A. Hillgren. 2012. 'Agonistic participatory design: working with marginalised social movements.' CoDesign: International Journal of CoCreation in Design and the Arts 8 (2-3): 127-144.

Botero, A. and S. Hyysalo. 2013. 'Ageing together: Steps towards evolutionary co-design in everyday practices.' CoDesign: International Journal of CoCreation in Design and the Arts 9 (1): 37-54.

Branco, R. M., J. Quental, and Ó. Ribeiro. 2017. 'Personalised participation: an approach to involve people with dementia and their families in a participatory design project.' CoDesign: International Journal of CoCreation in Design and the Arts 13 (2): $127-$ 143.

Brandt, E., T. Binder, and E. B. N. Sanders. 2012. 'Ways to Engage: Telling, Making and Enacting.' In Routledge International Handbook of Participatory Design, edited by J. Simonsen and T. Robertson, 145-181. New York: Routledge.

Braun, V., and V. Clarke. 2006. 'Using thematic analysis in psychology.' Qualitative Research in Psychology. 3 (2): 77-101.

Broadley, C., K. Champion, M. P. Johnson, and L. S. McHattie. 2016. 'From Participation to Collaboration: Reflections on the Co-Creation of Innovative Business Ideas.' Proceedings of The 50th Anniversary Design Research Society (DRS) Conference. 5: 1739-1758.

Chamberlain, A., A. Crabtree, and M. Davies. 2013. 'Community engagement for research: contextual design in rural CSCW system development.' Proceedings of the 6th International Conference on Communities and Technologies: 131-139.

Chevalier, J. M., and D. Buckles. 2013. Participatory Action Research: Theory and methods for Engaged Inquiry. London: Routledge.

Cruickshank, L., G. Coupe, and D. Hennesy. 2013. 'Beyond the castle:public space co-design, a case study and guidelines for designers'. Swedish Design Research Journal. 2: 48-57. 
DiSalvo, C., A. Clement, and V. Pipek. 2012. 'Participatory Design for, with, and by communities.' In Routledge International Handbook of Participatory Design, edited by J. Simonsen and T. Robertson, 182-209. New York: Routledge.

Dorst, K. 2015. Frame Innovation: Create New Thinking by Design. Cambridge, MA: MIT Press.

Development Trusts Association Scotland. 2017. What is a Development Trust? . http://www.dtascot.org.uk/content/what-is-a-development-trust

Ehn, P. 2008. 'Participation in Design Things.' Proceedings of the 2008 Conference on Participatory Design: 92-101.

Emilson, A., P. A. Hillgren, and A. Seravalli. 2014. 'Designing in the Neighborhood: Beyond (and in the Shadow of) Creative Communities.' In Making Futures: Marginal Notes on Innovation, Design, and Democracy, edited by P. Ehn, E. M. Nilsson, and R. Topgaard, 35-61. Cambridge, M. A: MIT Press.

Fox, S., and C. Le Dantec. 2014. 'Community historians: scaffolding community engagement through culture and heritage.' Proceedings of the 2014 conference on Designing interactive systems: 785-794.

Gaver, B., T. Dunne, and E. Pacenti. 1999. 'Design: Cultural Probes.' Interactions 6 (1): 21-29.

Gaver, B., A. Boucher, S. Pennington, and B. Walker. 2004. 'Cultural probes and the Value of Uncertainty.' Interactions 11 (5): 53-56.

Halse, J., and L. Boffi. 2014. 'Design interventions as a form of inquiry.' Paper presented at Research Network for Design Anthropology: Ethnographies of the Possible, Aarhus, $10^{\text {th }}-11^{\text {th }}$ April 2014.

Hanington, B. 2003. 'Methods in the Making: A Perspective on the State of Human Research in Design.’ Design Issues 19 (4): 9-18.

Hanington, B., and B. Martin. 2012. Universal Methods of Design: 100 ways to Research Complex Problems, Develop Innovative Ideas, and Design Effective Solutions. Beverley, MA: Rockport. 
Howard, Z., and M. M. Somerville. 2014. 'A comparative study of two design charrettes: implications for codesign and participatory action research.' CoDesign: International Journal of CoCreation in Design and the Arts 10 (1): 46-62.

IDEO. 2002. IDEO Method Cards. http://www.ideo.com/work/ method-cards/

Leapfrog. 2017a. About. http://leapfrog.tools/about/

Leapfrog, 2017b. Event Canvas. http://leapfrog.tools/tool/event-canvas/

Manzini, E., and F. Rizzo. 2011. 'Small projects/large changes: Participatory design as an open participated process.' CoDesign: International Journal of CoCreation in Design and the Arts 7 (3-4): 199-215.

Manzini, E. 2015. Design, When Everybody Designs: An Introduction to Design for Social Innovation. Cambridge, M. A: MIT Press

Mattelmäki, T. 2006. Design Probes. Helsinki: University of Art and Design Helsinki. http://www.uiah.fi/ publications.

Munoz, S. A., J. Farmer, J. Warburton, and J. Hall. 2014. 'Involving rural older people in service co-production: is there an untapped pool of potential participants?.' Journal of rural studies 34: 212-222.

Robertson, T., and I. Wagner. 2012. 'Engagement, Representation and Politics-in-action.’ In Routledge International Handbook of Participatory Design, edited by J. Simonsen and T. Robertson, 64-85. New York: Routledge.

Robertson, T., and J. Simonsen, eds. 2012. Routledge International Handbook of Participatory Design. New York: Routledge.

Sabiescu, A. G., S. David, I. van Zyl, and L. Cantoni. 2014. 'Emerging spaces in communitybased participatory design: reflections from two case studies.' Proceedings of the 13th Participatory Design Conference: 1-10. 
Sanders, E. B. N and P. Jan Stappers. 2008. 'Co-creation and the new landscapes of design.' CoDesign: International Journal of CoCreation in Design and the Arts 4 (1): 5-8.

Schön, D. A. 1983. The Reflective Practitioner: How Professionals Think in Action. Aldershot: Arena.

Smith, P. and C. Broadley. 2016. 'Contextualising Citizens: Design-Led Approaches To Visualising Community Ecologies, Building Interventions And Mobilising Citizen Participation.' Proceedings of MEDIATIONS: Art \& Design Agency and Participation in Public Space: 83-99.

Ssozi-Mugarura, F., E. Blake, and U. Rivett. 2017. 'Codesigning with communities to support rural water management in Uganda.' CoDesign: International Journal of CoCreation in Design and the Arts 13 (2): 110-126.

Steen, M. 2011. 'Tensions in human-centred design.' CoDesign: International Journal of CoCreation in Design and the Arts 7 (1): 45-60.

Steen, M. 2013. 'Virtues in Participatory Design: Cooperation, Curiosity, Creativity, Empowerment and Reflexivity.' Science and Engineering Ethics 19 (3); 945-962.

Vaajakallio, K. 2009. 'Enacting Design: understanding co-design as embodied practice.' Paper presented at Nordes 2009 - Engaging Artefacts, Oslo, 30 ${ }^{\text {th }}$ August $-1^{\text {st }}$ September 2009.

Vines, J., R. Clarke, P. Wright, J. McCarthy, and P. Oliver. 2013. 'Configuring Participation: On How We Involve People in Design.' Proceedings of the SIGCHI Conference on Human Factors in Computing Systems: 429-438.

Vestergaard, L., B. Hauge, and C. T. Hansen. 2016. 'Almost like being there; the power of personas when designing for foreign cultures.' CoDesign: International Journal of CoCreation in Design and the Arts 12 (4): 257-274.

Wang, D. and I. Oygur. 2010. 'A Heuristic Structure for Collaborative Design.' The Design Journal 13 (3)): 355-371. 
Winschiers-Theophilus, H., N. J. Bidwell, and E.Blake. 2012. 'Altering participation through interactions and reflections in design.' CoDesign: International Journal of CoCreation in Design and the Arts 8 (2-3): 163-182. 